\title{
Osteoporosis as an initial manifestation in a patient with Crohn's disease: A case report
}

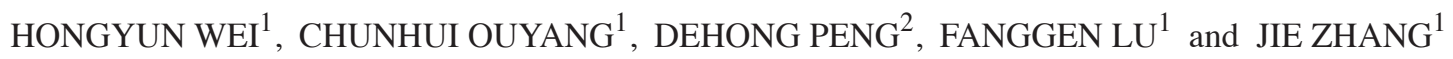 \\ Departments of ${ }^{1}$ Gastroenterology and ${ }^{2}$ Radiology, The Second Xiangya Hospital of \\ Central South University, Changsha, Hunan 410011, P.R. China
}

Received April 20, 2017; Accepted November 29, 2017

DOI: $10.3892 /$ etm.2018.5910

\begin{abstract}
The present case report details a rare case of osteoporosis as the initial manifestation of Crohn's disease (CD). A 43-year-old male was referred to the Second Xiangya Hospital of Central South University (Changsha, China) for further examination of low back pain (LBP) without digestive symptoms. Bone mineral density (BMD) analysis indicated osteoporosis, particularly in the lumbar spine. Endoscopy revealed an inflamed and strictured ileocecal valve with less inflammation in the ascending, transverse colon, sigmoid colon and rectum, compatible with $\mathrm{CD}$, which was in accordance with the appearance of an abdominal computed tomography scan. Duodenal-balloon enteroscopy indicated segmental ulceration and stricture in the jejunum, in accordance with CD. The patient was diagnosed with $\mathrm{CD}$ following examination. It was suspected that osteoporosis may be an extra-intestinal manifestation of CD. Steroids and biological agents were prescribed in sequence. LBP and BMD rapidly improved following treatment, and inflammatory markers returned to normal after 1.5 years of treatment. According to this case, osteoporosis with unknown causes should be considered as a possible sign of small intestinal CD.
\end{abstract}

\section{Introduction}

Bone loss is considered to be a disabling complication of inflammatory bowel disease (IBD) (1). Bone mineral density (BMD) testing results of patients with IBD from seven medical facilities, ranging from January 1996 to October 2006, were reviewed to determine the prevalence rate of osteoporosis (2). A total of 317 bone density tests were conducted in 2,035 patients, and osteoporosis was detected in $26 \%$ of patients and osteopenia in $48 \%$ (2). Another study involving 70 patients with

Correspondence to: Dr Jie Zhang, Department of Gastroenterology, The Second Xiangya Hospital of Central South University, 139 Remin Road, Changsha, Hunan 410011, P.R. China

E-mail: jiezhang@csu.edu.cn

Key words: Crohn's disease, bone density, osteoporosis, initial manifestation
IBD demonstrated that osteoporosis was observed in $13.2 \%$ of patients and osteopenia in $46.1 \%$ (3). The dominant risk factors leading to osteoporosis in IBD are considered to be age, intestinal malabsorption, long-term use of steroids, lack of exercise or supplementation of calcium and vitamin D, and smoking (4). In addition, inflammation and proinflammatory cytokines serve a key role in bone loss and increase the risk of fracture (5), including interleukin (IL)-6 and tumor necrosis factor (TNF) (6). However, the published literature regarding osteoporosis in IBD has associated it with the aforementioned risk factors. Osteoporosis as a complication is common in patients with IBD, followed by the above risk factors, while it rarely presents as a primary manifestation in patients with Crohn's disease (CD). A previous study observed that severe osteoporosis presented in a 12-year-old boy with $\mathrm{CD}$, and no history of steroid use was tracked (7). To the best of our knowledge, no reports of osteoporosis as an initial manifestation of IBD in adults exist. The present case report describes a case of osteoporosis as an initial manifestation in an adult patient with CD without the aforementioned risk factors. Notably, using steroids and biological agents ameliorated low back pain (LBP) in the patient. Considering the relationship between inflammation and bone loss, it was hypothesized that osteoporosis, in the present case, is a primary manifestation of CD.

\section{Case report}

A 43-year-old male presented at the Second Xiangya Hospital of Central South University (Changsha, China) with worsening symptoms of LBP in July 2015 and was compulsively posed in a lateral position. No abdominal or digestive syndromes were observed. The dietary intake of the patient had not changed; however, the patient had lost $15 \mathrm{~kg}$ of body weight in 12 months. As demonstrated in Fig. 1, X-rays revealed thoracolumbar spine degeneration (Fig. 1A). A 1 year supply of medicinal calcium preparation (Caltrate ${ }^{\circledR} ; 600 \mathrm{mg} /$ day orally; Wyeth; Pfizer, Inc., New York, NY, USA; and $1 \alpha-\mathrm{OH}$ vitamin D3; Teva Pharmaceutical Industries, Ltd., Petach Tikva, Israel; oral soft capsule, $0.25 \mu \mathrm{g} /$ day) was unable to improve LBP. A computed tomography (CT) scan was subsequently performed, revealing thoracolumbar spine degeneration and mesenteric lymph node tumescence (Fig. 1B), as did single photon emission CT (Fig. 1C). The patient was therefore referred to the Gastroenterology department in July 2015. 
The patient had no history of long-term use of corticosteroids and no bone fractures, and had a 10-year history of cigarette smoking at a rate of 6 cigarettes/day. The body weight of the patient was $71.6 \mathrm{~kg}$ and body mass index was 23.92. Physical examination revealed pressing pain in the cervical, lumbar and thoracic vertebrae and obvious clubbing of the fingers and feet (Fig. 1D). Other inspections of the lung, heart and abdomen proved unremarkable. Laboratory data revealed a serum hemoglobin $(\mathrm{Hb})$ level of $103 \mathrm{~g} / 1$ (normal range, 130-175 g/l; Sysmex ${ }^{\circledR}$ XN-Series; Sysmex Corporation, Kobe, Japan), serum albumin level of $23.9 \mathrm{~g} / 1$ (normal range, 40-55 g/1; Hitachi Modular 7600 chemistry analyzer; Hitachi, Ltd., Tokyo, Japan), an erythrocyte sedimentation rate $(\mathrm{ESR})$ of $50 \mathrm{~mm} / \mathrm{h}$ (normal range, $1-15 \mathrm{~mm} / \mathrm{h}$, automatic ESR analyzer Monitor-100; Vital Diagnostics, Forli, Italy), C-reactive protein (CRP) level of $63.5 \mathrm{mg} / 1$ (normal range, 0-8.0 mg/1; IMMAGE ${ }^{\circledR}$ 800; Beckman Coulter, Inc., Brea, CA, USA) and reduced serum 25-hydroxy vitamin D level of $37 \mathrm{nmol} / \mathrm{l}$ (normal range, 75-250 nmol/l; ADVIA Centaur XP chemiluminescence immunoanalyzer; Siemens Healthcare Diagnostics Manufacturing, Ltd., Dublin, Ireland). No positive results were identified for serum parathyrin, gonadin, calcium, phosphate, magnesium, alkaline phosphatase and human leukocyte antigen-B27.

A dual-energy X-ray absorptionmetry scan (DXA) of the lumbar spine revealed a severe reduction in BMD (lumbar Z-score, -2 .9 SD; T-score, -3.0 SD). Colonoscopy demonstrated an inflamed and strictured ileocecal valve, with less inflammation in the ascending, transverse colon, sigmoid colon and rectum, compatible with $\mathrm{CD}$; however, the coloscope could not pass through the restricted ileocecal valve (Fig. 1E and F). An ileocecal valve biopsy indicated chronic active colitis with ulcers and inflammatory granulation tissue suggestive of $\mathrm{CD}$ (Fig. $1 \mathrm{G}$ and $\mathrm{H}$ ). The biopsy tissue was fixed in $4 \%$ formaldehyde for $6 \mathrm{~h}$ at $35^{\circ} \mathrm{C}$, then cut into 4- $\mu$ m-thick sections and stained with hematoxylin and eosin for $20 \mathrm{~min}$ at room temperature. The sections were observed using an Olympus BX53F light microscope (Olympus Corporation, Tokyo, Japan). An abdominal CT scan indicated marked symmetric segmental thickening at the jejunum wall (Fig. 2A and B). Duodenal-balloon enteroscopy demonstrated segmental ulceration and stricture in the jejunum (Fig. 2C). A jejunum biopsy revealed chronic active colitis with necrosis and ulceration (Figs. 1I and 2D). The tissue sections were fixed and stained as above. After excluding tuberculosis via a chest X-ray and T-SPOT ${ }^{\circledR}$ (cat. no. TB.300 CN; Oxford Immunotec Global PLC, Abingdon, UK), the patient was diagnosed with CD according to World Health Organization criteria (8). The presenting CD activity index (CDAI) was 231.0 (9).

Prednisone (Zhejiang Xianju Pharmaceutical Co., Ltd., Zhejiang, China) $60 \mathrm{mg} /$ day was prescribed with a concomitant dose of $100 \mathrm{mg}$ azathioprim (Shanghai Xinyi Pharmaceutical Co., Ltd., Shanghai, China) daily in September 2015 for 8 months. LBP began to show improvement 5 days after treatment. However, by April 2016, the CRP level was $57.60 \mathrm{mg} / \mathrm{l}$, the CDAI was 284, and a second DXA revealed no improvement of BMD (lumbar Z-score, -3.1 SD; T-score, -3.2 SD). In addition, hypertension, an adverse effect of prednisone, was tracked. Given this result and the patient's insensitivity to steroids, prednisone was switched to adalimumab (Humira ${ }^{\circledR}$;
$40 \mathrm{mg} / 2$ weeks, subcutaneous injection; Vetter Pharma International GmbH, Ravensburg, Germany) in May 2016. By November 2016, CRP and ESR were maintained at normal levels (7.12 mg/l and $10 \mathrm{~mm} / \mathrm{h}$, respectively), Hb level was $158 \mathrm{~g} / \mathrm{l}$ and the CDAI was 148.0. DXA revealed an improved BMD (lumbar Z score, -1.9 SD; T-score, -2.1 SD) and abdominal CT scan demonstrated improved mineralization, with sclerosis of vertebral bodies and intestinal inflammation (Fig. 2E). Nevertheless, capsule endoscopy indicated no significant improvement of small intestinal disease (Fig. 2F). The patient is still undergoing treatment with biological agents and is followed up every 2 months. The patient provided written informed consent for publication of the present case report.

\section{Discussion}

Reduced bone density is an extra-intestinal manifestation and a common complication of IBD (10). However, osteoporosis as an initial symptom of IBD is rare. The present report detailed a case of osteoporosis as a presenting manifestation of $\mathrm{CD}$, and osteoporosis was improved following treatment of $\mathrm{CD}$ with glucocorticoids.

The possible reasons leading to osteoporosis in IBD are corticosteroid use, smoking and gut inflammation (10). No history of corticosteroid use and metabolic bone disease were tracked in the present patient. Smoking alone did not suitably explain osteoporosis. Therefore, the inflammatory activity of $\mathrm{CD}$ itself may be a main contributor to osteoporosis (11). One of the possible mechanisms of osteoposrosis in IBD is malabsorption. Calcium deficiency and low vitamin D levels occurred as a result of small intestinal dysfunction in the present patient. A previous 5-year study identified that low vitamin D levels are common in patients with IBD, and IBD patients with low mean vitamin D levels demonstrated worse disease activity, worse pain and higher requirement for steroids (4). The use of vitamin D may improve osteoporosis (12); however, this was not the case for the present patient. LBP was not improved following treatment with calcium and vitamin D. Furthermore, although BMD improved following steroid and biological treatment, endoscopic performance was not notably improved in the present case. This phenomenon suggested that malabsorption may be a partial cause of osteoporosis in CD, and osteoporosis may be the result of extra-intestinal inflammation.

In the present case, prednisone and azathioprim were prescribed, and the LBP began to improve following treatment, indicating that osteoporosis was associated with inflammation. Immune-associated inflammation also participated in the genesis of bone loss, as previously reported (13). T cell reconstitution is accompanied by increased bone resorption and decreased BMD (14). Proinflammatory cytokines may also contribute to bone loss and increase the risk of fracture (5). A previous study demonstrated that IL-6 levels were higher in patients with $\mathrm{CD}$ than the levels in controls (15). Thus, steroids were able to reduce LBP in the present case. Nevertheless, the present patient suffered from adverse effects and insensitivity to steroids, so adalimumab, an anti-TNF antibody, was administered, which resulted in improved BMD and CDAI. TNFs are cytokines that are associated with bone loss, and the pathway involved is the receptor activator of nuclear factor- $\kappa \mathrm{B}$, which 

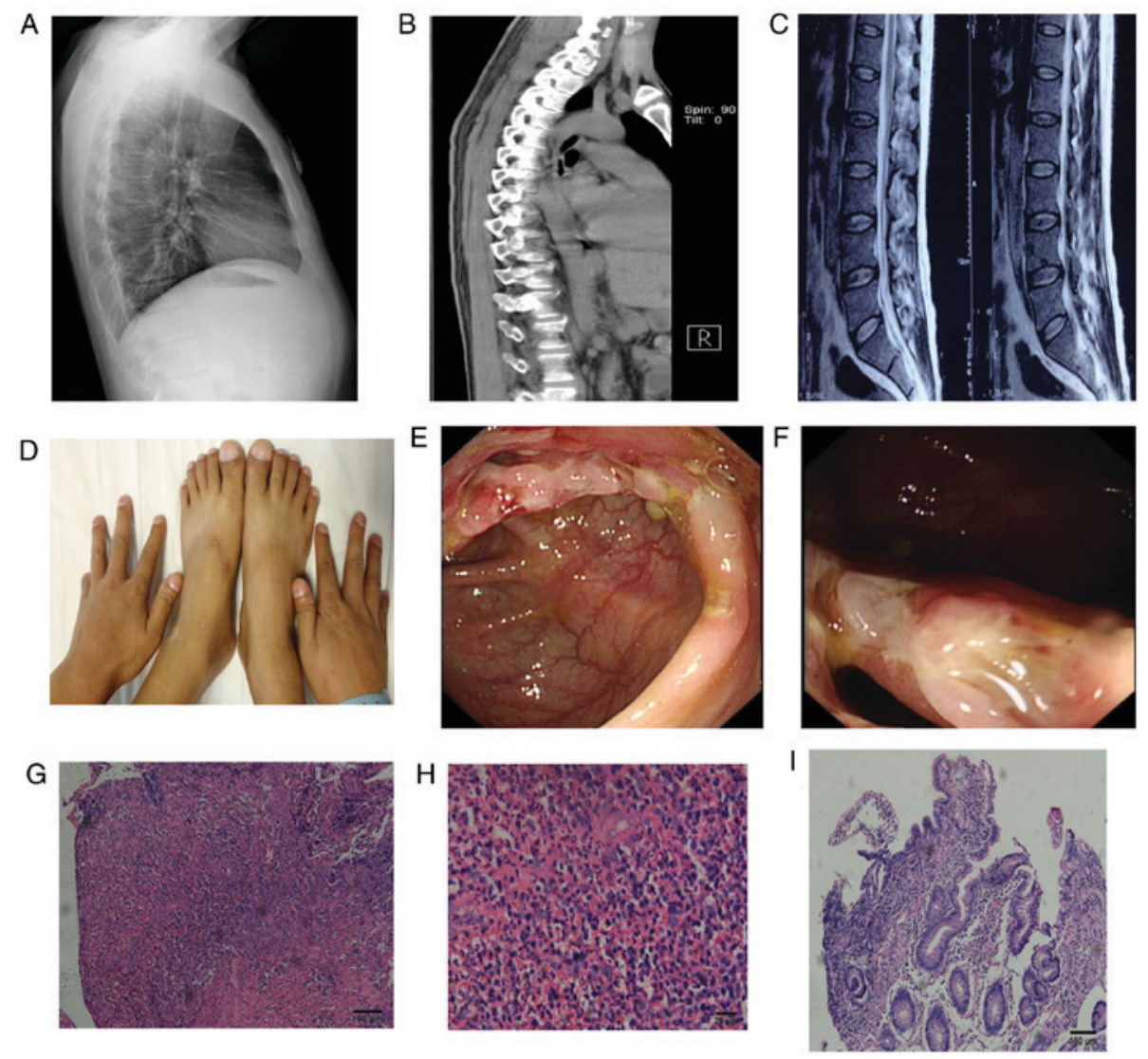

Figure 1. Examination of bone and intestinal manifestation under colonic endoscopy. (A-C) Bone loss was identified following radical examination. (D) The patient demonstrated clubbing of the fingers and feet. ( $\mathrm{E}$ and F) Colonoscopy indicated an inflamed and strictured ileocecal valve. ( $\mathrm{G}$ and $\mathrm{H}$ ) Biopsy under colonoscopy revealed chronic active colitis with ulcers and inflammatory granulation tissue suggestive of Crohn's disease (G, magnification, x100; $\mathrm{H}$, magnification, $x 400$ ). (I) Biopsy under duodenal-balloon enteroscopy demonstrated chronic active colitis with necrosis and ulceration in the small intestine (magnification, x100).
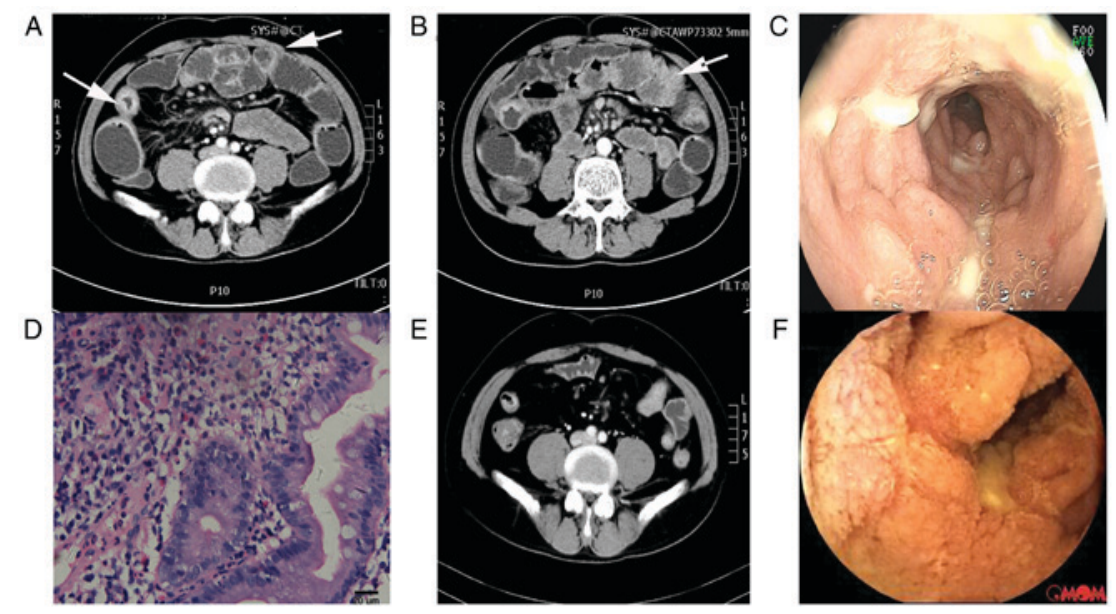

Figure 2. CT scan and endoscopy examination prior to and following treatment. (A and B) CT demonstrated marked symmetric segmental thickening at the jejunum wall prior to treatment (white arrows). (C) Segmental ulceration and stricture in the jejunum under duodenal-balloon enteroscopy was observed and (D) chronic active colitis with ulceration was revealed under the microscope (magnification, $\mathrm{x} 400$ ) prior to treatment. (E) Reduced mesenteric lymph node under CT and (F) improved intestinal inflammation under capsule endoscopy was observed following treatment. CT, computed tomography.

is expressed on the surface of osteoblasts (16). Additionally, interferon regulatory factor-1 (IRF1) is regarded as a genetic risk for IBD, and a mutant murine model of IRF1 $1^{-/}$indicated that IRF1 deficiency was related to decreased proliferation of bone marrow-derived osteoblast precursors and increased mineralization activity, suggesting its role in regulating bone metabolism (17). Accordingly, bone loss may be an initial manifestation of $\mathrm{CD}$.

In conclusion, for bone loss, the possibility of small intestinal CD should be considered, and CD as the primary disease should be treated actively. Adequate calcium supplements should be administered to patients with $\mathrm{CD}$, physical activity 
should be encouraged, and smoking or excessive alcoholic intake should also be avoided.

\section{Acknowledgements}

Not applicable.

\section{Funding}

No funding was received.

\section{Availability of data and materials}

The datasets used and/or analyzed during the current study are available from the corresponding author on reasonable request.

\section{Authors' contributions}

CO performed endoscopy examination. DP performed the magnetic resonance imaging and computed tomography scans. FL and HW collected the data and wrote the manuscript. JZ analyzed the data and revised the manuscript.

\section{Ethics approval and consent to participate}

The patient provided written informed consent for the publication of this case report.

\section{Consent for publication}

The patient provided written informed consent for the publication of any associated data and accompanying images.

\section{Competing interests}

The authors declare that they have no competing interests.

\section{References}

1. Ott $\mathrm{C}$ and Schölmerich J: Extraintestinal manifestations and complications in IBD. Nat Rev Gastroenterol Hepatol 10: 585-595, 2013.

2. Etzel JP, Larson MF, Anawalt BD, Collins J and Dominitz JA Assessment and management of low bone density in inflammatory bowel disease and performance of professional society guidelines. Inflamm Bowel Dis 17: 2122-2129, 2011.
3. Miznerova E, Hlavaty T, KollerT, Toth J, Holociova K, Huorka M, Killinger $\mathrm{Z}$ and Payer J: The prevalence and risk factors for osteoporosis in patients with inflammatory bowel disease. Bratisl Lek Listy 114: 439-445, 2013.

4. Kabbani TA, Koutroubakis IE, Schoen RE, Ramos-Rivers C, Shah N, Swoger J, Regueiro M, Barrie A, Schwartz M, Hashash JG, et al: Association of vitamin D level with clinical status in inflammatory bowel disease: A 5-year longitudinal study. Am J Gastroenterol 111: 712-719, 2016.

5. Clowes JA, Riggs BL and Khosla S: The role of the immune system in the pathophysiology of osteoporosis. Immunol Rev 208: 207-227, 2005.

6. Schulte CM, Dignass AU, Goebell H, Röher HD and Schulte KM: Genetic factors determine extent of bone loss in inflammatory bowel disease. Gastroenterology 119: 909-920, 2000.

7. Thearle M, Horlick M, Bilezikian JP, Levy J, Gertner JM, Levine LS, Harbison M, Berdon $\mathrm{W}$ and Oberfield SE: Osteoporosis: An unusual presentation of childhood Crohn's disease. J Clin Endocrinol Metab 85: 2122-2126, 2000.

8. APDW2004 Chinese IBD Working Group: Retrospective analysis of 515 cases of Crohn's disease hospitalization in China: Nationwide study from 1990 to 2003. J Gastroenterol Hepatol 21: 1009-1015, 2006.

9. Harvey RF and Bradshaw JM: A simple index of Crohn's-disease activity. Lancet 1: 514, 1980.

10. Targownik LE, Bernstein CN and Leslie WD: Inflammatory bowel disease and the risk of osteoporosis and fracture. Maturitas 76: 315-319, 2013.

11. Phan CM and Guglielmi G: Metabolic bone disease in patients with malabsorption. Semin Musculoskelet Radiol 20: 369-375, 2016.

12. Lee DY, Jee JH, Cho YY, Jang JY, Yu TY, Kim TH, Hong YJ, Hong WJ, Jin SM, Hur KY, et al: Serum 25-hydroxyvitamin D cutoffs for functional bone measures in postmenopausal osteoporosis. Osteoporos Int 28: 1377-1384, 2017.

13. Iseme RA, McEvoy M, Kelly B, Agnew L, Walker FR and Attia J: Is osteoporosis an autoimmune mediated disorder? Bone Rep 7: 121-131, 2017.

14. Ofotokun I, Titanji K, Vikulina T, Roser-Page S, Yamaguchi M, Zayzafoon M, Williams IR and Weitzmann MN: Role of T-cell reconstitution in HIV-1 antiretroviral therapy-induced bone loss. Nat Commun 6: 8282, 2015.

15. Sylvester FA, Wyzga N, Hyams JS and Gronowicz GA: Effect of Crohn's disease on bone metabolism in vitro: A role for interleukin-6. J Bone Miner Res 17: 695-702, 2002.

16. Ali T, Lam D, Bronze MS and Humphrey MB: Osteoporosis in inflammatory bowel disease. Am J Med 122: 599-604, 2009.

17. Salem S, Gao C, Li A, Wang H, Nguyen-Yamamoto L, Goltzman D, Henderson JE and Gros P: A novel role for interferon regulatory factor 1 (IRF1) in regulation of bone metabolism. J Cell Mol Med 18: 1588-1598, 2014. 